[Reprinted from the Journal of Physical Chemistry, 56, 475 (1952).]

\title{
On the Kinetics of Evaporation
}

By S. S. Penner 


\title{
ON THE KINETICS OF EVAPORATION
}

\author{
By S. S. Penner \\ Guggenheim Jet Propulsion Center, California Institute of Technology, Pasadena, California \\ Received May 9, 1951
}

\begin{abstract}
The kinetics of evaporation of liquids has been reconsidered from the point of view of classical reaction kinetics and also by application of the theory of absolute reaction rates. It is shown that evaporation treated as a unimolecular rate process, with a rate proportional to the surface concentration of energetic molecules, leads to the Knudsen equation for spherical molecules provided 6 square terms contribute to the energy of activation. As was pointed out in an earlier publication, the theory of absolute reaction rates, after correction for lack of equilibrium between normal molecules and the activated complex, leads to the Knudsen equation for spherically symmetric molecules if reasonable assumptions are made concerning the nature of the activated complex. Evidence is presented in support of the idea that the equilibrium theory of absolute reaction rates is not consistent with the model of the liquid used to determine evaporation rates. The theoretical treatment is next extended to polar liquids with restricted rotation and it is shown that the evaporation coefficient should be identified with the free-angle ratio, a conclusion which has been verified quantitatively by Wyllie. ${ }^{1}$
\end{abstract}

\section{Introduction}

The theory of absolute reaction rates ${ }^{2}$ has been applied in previous publications to an analysis of the kinetics of evaporation of liquids, ${ }^{3}$ which has also been examined by straightforward applications of kinetic theory ${ }^{4}$ and elementary reaction kinetics. ${ }^{5}$ The analysis using the theory of absolute reaction rates has been based on the free-volume model of the liquid rather than on the more adequate description of the liquid state developed by Kirkwood $^{6}$ and by Born and Green. ${ }^{7}$ By introducing the hypothesis that the activated state formed during evaporation corresponds to a molecule moving freely in an area equal to the two-thirds power of the volume per molecule in the liquid, it was shown that a relation of the same form as the Knudsen equation is obtained for the specific evaporation rate. Here it was assumed that the rotational and vibrational partition functions of the liquid molecules and of the activated complex are the same. ${ }^{3}$ Furthermore, the specific evaporation rate was shown to be practically identical with the Knudsen equation (with a transmission coefficient replacing the empirically observed evaporation coefficient ${ }^{8}$ ) after a suitable correction, suggested by the nonequilibrium theory of absolute reaction rates, ${ }^{9}$ had been introduced. Thus it has been shown that absolute reaction rate theory, together with a simplified model of the liquid state, can be used to derive a relation for the evaporation rate which is known to have the correct functional form.

The treatment given previously applies only to liquids with spherically symmetric force fields which allow free rotation. For pure liquids of this type the evaporation coefficient is known to be

(1) G. Wyllie, Proc. Roy. Soc. (London), 197A, 383 (1949).

(2) S. Glasstone, K. J. Laidler, and H. Eyring. "The Theory of Rate Processes," MeGraw-Hill Book Co., Ine., New York, N. Y., 1941.

(3) S. S. Penner, Thrs Journal, 52, 950 (1948); ibid., 52, 1262 (1948).

(4) J. Frenkel, "Kinetic Theory of Liquids," Clarendon Press, Oxford, 1946, Chap. I; $Z$ Physik, 26, 117 (1924).

(5) S. S. Penner, This Jotrnal, 52, 368 (1948)

(6) J. G. Kirkwood, et al., J. Chem. Phys., 18, 1040 (1950), and earlier publications.

(7) M. Born and H. S. Green, "A General Kinetic Theory of Liquids," Cambridge University Press, Cambridge, 1949; also Proc. Roy. Soc. (London), Volumes 188A to 194A.

(8) The evaporation coefficient is defined as the ratio of the observed rate of evaporation to the evaporation rate calculated from the Knudsen equation.

(9) J. O. Hirsehfelder, J. Chem. Phys., 16, 22 (1948) close to unity, ${ }^{10}$ whence it follows that the transmission coefficient must also be equal to unity in order to obtain agreement between experimental data and the result derived from the theory of absolute reaction rates.

Recently Wyllie ${ }^{\mathrm{T}}$ has called attention to an interesting quantitative correlation between the evaporation coefficient for polar liquids and the free-angle ratio introduced by Kincaid and Eyring ${ }^{11}$ to allow for restricted rotation in the liquid. We shall show that this result is a direct consequence of our simplified rate theory when applied to polar liquids.

Before presenting the extension of the simplified theory for the kinetics of evaporation to polar liquids, we shall reconsider the problems of evaporation rates from the point of view of classical reaction kinetics in order to amplify some of the numerical calculations described previously. ${ }^{5}$ In particular, it will be shown that evaporation treated as a unimolecular process, with the evaporation rate proportional to the concentration of energetic molecules at the surface, will lead to the Knudsen equation if it is assumed that 6 square terms contribute to the activation energy. ${ }^{12}$ Finally, the problem of applicability of the non-equilibrium theory of absolute reaction rates is reconsidered. It is shown that the assumption of equilibrium between normal molecules and the activated complex leads to the . requirement of an abnormally long energy barrier, thereby suggesting that equilibrium rate theory is not applicable to the problem at hand. Compelling reasons for using the particular non-equilibrium model considered by Hirschfelder ${ }^{9}$ are, however, not apparent.

\section{Evaporation Rates as a Problem in Classical Chemical Kinetics}

A treatment of evaporation rates based on classical reaction kinetics requires the assumption of a specific mechanism for evaporation. Since vaporization is a surface phenomenon, it seems reasonable to suppose that the rate of loss of molecules $-\mathrm{d} n_{\mathrm{v}}$ f $\mathrm{d} t$ from a given volume $V$ is proportional to the number of molecules $n_{\mathrm{s}}$ exposed at the surface, i.e.

(10) E. H, Kennard, "Kinetic Theory of Gases," MeGraw-Hill Book Co., Inc., New York, N. Y., 1938, pp. 68-71.

(11) J. F. Kincaid and H. Eyring, J. Chem. Phys., 6, 620 (1938).

(12) L. Kassel, "Kineties of Homogeneous Gas Reaction," Chemical Catalog Company, (Reinhold Publ. Corp.), New York, N. Y., 1932, Chapter V. 


$$
-\mathrm{d} n_{\mathrm{V}} / \mathrm{d} t=j_{\mathrm{e}} n_{\mathrm{s}}
$$

where $j_{\mathrm{e}}$ is a rate constant for evaporation. Since $n_{\mathrm{V}}=n V$ and $n_{\mathrm{s}}=n^{2 / 3} S$, where $n$ is the number of molecules per unit volume and $S$ is the surface area of the evaporating compound, it follows that

$$
\begin{gathered}
-\rho n \mathrm{~d} V / \mathrm{d} t=\mu j_{\mathrm{e}} n^{2} / \Omega S \\
\text { or } \\
G=-\rho(1 / S)(\mathrm{d} V / \mathrm{d} t)=j_{\mathrm{e}} \rho / n^{1 / 3}
\end{gathered}
$$

Here $\rho$ is the density of the evaporating compound and $G$, the specific evaporation rate, represents the rate at which mass is lost by evaporation per unit time per unit surface area.

On the basis of elementary considerations in chemical reaction kinetics it seems intuitively obvious to equate $j_{\mathrm{e}}$ to the product of a frequency factor whose dimensions are sec. ${ }^{-1}$ and an exponential factor involving an appropriate activation energy to single out the fraction of molecules with sufficient energy for evaporation. Therefore we may write

$$
j_{\mathrm{e}}=B \exp \left(-\Delta E_{\mathrm{v}} / R T\right)
$$

where $\Delta E_{\mathrm{V}}$ is the molar activation energy for evaporation, $R$ is the molar gas constant, $T$ is the absolute temperature, and $B$ is the as yet undetermined frequency factor. The term $\exp \left(-\Delta E_{\mathrm{V}} / R T\right)$ in equation 3 arises because only the molecules with energy in excess of $\Delta E_{\mathrm{V}}$ per mole can evaporate, and the fraction of molecules possessing the required energy at any given time is $\exp \left(-\Delta E_{\mathrm{V}} / R T\right)$. The molar activation energy for evaporation is related to the heat of evaporation $\Delta H_{\mathrm{V}}$ according to the well-known relation ${ }^{2}$

$$
\Delta H_{\mathrm{V}}=\Delta E_{\mathrm{V}}+N p_{\mathrm{s}}\left(v_{\mathrm{g}}-v_{\mathrm{s}}\right)
$$

since the external work done during the expansion of one mole from the free volume per molecule $v_{f}$ in the condensed state to the volume per molecule $v_{\mathrm{g}}$ in the gaseous state at the vapor pressure $p_{\mathrm{s}}$ is $N p_{\mathrm{s}}\left(v_{\mathrm{g}}-v_{\mathrm{f}}\right)$ where $N$ represents the Avogadro number. But $v_{\mathrm{f}}$ is negligibly small compared to $v_{\mathrm{g}}$ whence $N p_{\mathrm{s}}\left(v_{\mathrm{g}}-v_{\mathrm{f}}\right) \simeq R T$ if the vapor behaves as a perfect gas. It therefore follows that, to a close approximation

$$
j_{\mathrm{e}}=e B \exp \left(-\Delta H_{\mathrm{v}} / R T\right)
$$

The evaluation of the frequency factor $B$ corresponding to an upper limit for the rate of evaporation can be carried out intuitively by noting that $B$ should represent some sort of an upper limit for the oscillation or collision frequency. An upper limit for the collision frequency of the evaporating molecules is given by the root mean square velocity of activated molecules divided by the mean free path. But the mean square velocity of activated molecules $C^{2}$ is simply the mean square velocity of molecules with energy in excess of $\Delta E \mathrm{~V}$ per mole. ${ }^{5}$ A simple calculation utilizing the Maxwell-Boltzmann law in two dimensions shows that $C^{2}$ is given by the relation

$$
C^{2}=\frac{1}{n_{\mathrm{s}} e^{-\Delta E \mathrm{v} /} R T} \int_{\Delta E \mathrm{v}}^{\infty} \frac{n_{\vartheta}}{R T} e^{-E / R T} \times \frac{2 E}{M} \mathrm{~d} E
$$

or

$$
C^{2}=(2 / M)\left(\Delta E_{\mathrm{V}}+R T\right)=\left(2 \Delta H_{\mathrm{V}} / M\right)
$$

where $M$ represents the molecular weight of the evaporating compound. Hence the frequency factor $B$ is

$$
B=\sqrt{C^{2}} / v_{\mathrm{f}}^{1 / 2}=\left(2 \Delta H_{\mathrm{V}} / M\right)^{1 / 2} / v_{\mathrm{f}}^{1 / 8}
$$

and, therefore,

$$
G=e \rho\left[\left(2 \Delta H_{\mathrm{V}} / M\right)^{1 / 2} / v_{\mathrm{f}}^{1 / 3} n^{1 / 3}\right] \exp \left(-\Delta H_{\mathrm{V}} / R T\right) \text { (6) }
$$

where the mean free path in the liquid has been set equal to the cube root of the free volume per molecule $v_{\mathrm{f}}$ in the condensed state. The free volume $v_{\mathrm{f}}$ for liquids can be calculated, for example, from the Kincaid-Eyring expression ${ }^{\mathbf{1 1}}$

$$
v_{\mathrm{f}}=v(1 / U)^{3}\left(R T_{\gamma} / M\right)^{3 / 2}
$$

where $v$ is the volume per molecule in the liquid state, $M$ is the molecular weight of the evaporating liquid, $U$ is the sound velocity in the liquid, and $\gamma$ is the ratio of the specific heat at constant pressure to the specific heat at constant volume. It has been shown that the use of equations 6 and 7 actually leads to results ${ }^{5}$ which are in some cases in good agreement with numerical values calculated from the Knudsen equation, i.e., with results calculated from the relation

$$
G=\epsilon p_{s}(M / 2 \pi R T)^{1 / 2}
$$

where the evaporation coefficient $\epsilon$ has been set equal to unity and $p_{\mathrm{s}}$ is the saturated vapor pressure of the evaporating compound. The numerical results calculated from equations 6 and 7 and from equation 8, respectively, are in fairly good agreement for compounds such as $\mathrm{CCl}_{4}, \mathrm{CHCl}_{3}, \mathrm{C}_{6} \mathrm{H}_{6}$, etc., for which the free volume model is a satisfactory description of the liquid state. For associated liquids such as water or alcohol $v_{\mathrm{f}}$ is not adequately represented by equation 7 and failure to obtain satisfactory agreement between the theoretical equations is therefore not surprising.

The equivalence of equations 6 and 8 can be demonstrated more explicitly by proceeding as follows. At room temperature $\Delta E_{\mathrm{V}}>>T$ for ordinary liquids. Therefore $(2 \Delta H \mathrm{v} / M)^{1 / 2} \simeq$ $\left(2 \Delta E_{\mathrm{V}} / M\right)^{1 / 2}$. Hence equation 6 can be written as

$$
G \simeq\left(2 \Delta E_{\mathrm{V}} / M\right)^{1 / 2}\left(1 / v_{i}\right)^{1 / 3}\left(\rho / n^{1 / 3}\right) e \exp \left(-\Delta H_{\mathrm{V}} / R T\right)
$$

But it has been shown ${ }^{2}$ that $\left(\Delta E_{\mathrm{V}} / R T\right)^{1 / 2} \simeq(2)^{1 / 2}$ $\left(v / v_{\mathrm{f}}\right)^{1 / 6}$ and, therefore

$$
G \simeq\left(\frac{2 k T}{m}\right)^{1 / 2} \sqrt{2}\left(v / v_{\mathrm{f}}\right)^{1 / 8} \frac{v_{\mathrm{f}}^{2 / 3}}{v_{\mathrm{f}}} \frac{\rho v^{1 / 3}}{n^{1 / 8} v^{1 / 3}} e \exp \left(-\Delta H_{\mathrm{V}} / R T\right)
$$

where $k$ is the Boltzmann constant and $m$ represents the mass per molecule. But $\rho v=m, n^{1 / 3} v^{1 / s}=1$ and

whence

$$
1 / v_{\mathrm{f}}=\left(p_{\mathrm{s}} / k T\right) e^{\Delta H \mathrm{v} / R T}
$$

$$
G \simeq p_{\mathrm{s}}(m / 2 \pi k T)^{1 / 2}\left[2 \sqrt{2 \pi} e\left(v_{\mathrm{f}} / v\right)^{1 / 2}\right]
$$

In equation $9 p_{\mathrm{s}}$ represents the saturated vapor pressure of the evaporating compound whose vapor is assumed to behave as a perfect gas. Equation 9 was obtained by Eyring and Hirschfelder ${ }^{13}$ who noted that the Gibbs free energy of the evaporating substance and of the gas with which it is in equilibrium must be equal to each other. ${ }^{14}$ Equation 10 is seen to be identical with the Knudsen equation 8

(13) H. Eyring and J. O. Hirschfelder, This Jodrnal, 41, 249 (1937)

(14) It should be noted that equation 9 holds only for spherically svmmetric liquid molecules. 
with $\epsilon=1$ except for the term in square brackets. Since $v_{\mathrm{f}} / v \simeq 3 \times 10^{-3}$ at room temperature for many liquids, the term in square brackets is of the order of unity. This result accounts for the agreement observed at room temperature between the numerical values calculated from equations 6 and 8 , respectively. However, since $v_{\mathrm{f}} / v$ varies with temperature, it is obvious that equations 8 and 10 do not have the same temperature dependence. This conclusion was reached previously as the result of empirical calculation. ${ }^{5}$

An equation equivalent to the Knudsen equation can be obtained by using some of the methods introduced into the classical theory of unimolecular decompositions ${ }^{12,15-17}$ in a rather arbitrary manner. On the basis of a simple collision mechanism it might be expected that the frequency factor in the rate expression for evaporation is of the same order of magnitude as the mean collision frequency, i.e.

$$
B=\overline{\dot{x}} / v_{\mathrm{f}}^{1 / \mathrm{s}}=(k T / 2 \pi m)^{1 / 2} / v_{\mathrm{f}}{ }^{1 / s}
$$

where $\overline{\dot{x}}$ is the mean translational velocity in a given direction of a molecule of mass $m$ at the temperature $T$. On the basis of the preceding discussion it is, of course, obvious that the use of equation 11 with the exponential factor $\exp \left(-\Delta E_{\mathrm{V}} / R T\right)$ will lead to low values for $G$. In order to obtain the Knudsen equation it is evidently necessary to increase the rate of production of energetic molecules. The desired result can be attained either by the arbitrary introduction of an entropy term or else by the equally arbitrary assumption that more than two square terms can contribute to the energy of activation, thus increasing the probability of the occurrence of energetic molecules.

It was pointed out a number of years ago that agreement with experimental results could be obtained for unimolecular decomposition if $n$ square terms can contribute to the energy of activation where $n$ appears to be related to the complexity of the decomposing molecule. ${ }^{15,16}$ If a number of restrictive conditions are met, the effective frequency factor is found to be larger than the collision number by the factor

$$
\left(\Delta E_{\mathrm{v}} / R T\right)^{(1 / 2) \mathrm{n}-1 /[(1 / 2) n-1] !}
$$

If the arbitrary assumption is made that 6 square terms can contribute to the energy of activation, then it can be seen that the effective frequency factor is

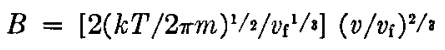

since

$$
\Delta E_{\mathrm{V}} / R T \simeq 2\left(v / v_{\mathrm{f}}\right)^{1 / \mathrm{s}}
$$

Equations 2, 3 and 13 lead to the relation

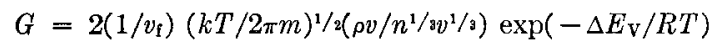

or

$$
G=2 e p_{\mathrm{s}}(m / 2 \pi k T)^{1 / 2}
$$

where use has been made of equation 9 as well as of the relations $\rho v=m$ and $n^{1 / 3} v^{2 / 3}=1$. Equation 14 is the Knudsen equation with an evaporation coefficient of unity and multiplied by the factor $2 e$.

(15) R. H. Fowler and E. K. Rideal, Proc. Roy. Soc. (London), 113A, 570 (1927)

(16) C. N Hinshelwood, ibid., 113A, 230 (1927).

(17) O. K. Rice and H. C. Ramsperger, J. Am. Chem. Soc., 49, 617 (1927); 50, $617(1928)$
On the basis of classical considerations it has thus proved to be possible to obtain a relation of the same form as the Knudsen equation by making use of an artifice which was introduced into the theoretical discussion of unimolecular decomposition prior to the advent of the theory of absolute reaction rates. The physical significance of the need for the assumption that 6 square terms can contribute to the energy of activation is obscure.

The classical considerations reviewed in the preceding discussion are not particularly convincing since they were designed to fit the desired results rather than the physical processes. A significant improvement over this type of argument can be registered through the application of the theory of absolute reaction rates as discussed in the following section.

\section{Rates of Evaporation from the Point of View of the Theory of Absolute Reaction Rates}

According to the statistical theory of reaction rates $^{2}$ the rate constant for evaporation $j_{\mathrm{e}}$ is given by the relation

$$
j_{\mathrm{e}}=K(k T / h)\left(Q^{*} / Q\right) \exp \left(-\Delta E_{\mathrm{V}} / R T\right)
$$

where $K$ is the transmission coefficient, $h$ represents Planck's constant, $Q^{*}$ is the partition function of the activated complex with the energy zero referred to the zero-point energy of the activated state and the translational partition function along the coordinate of decomposition removed and $Q$ is the complete partition function of the normal molecules with energy zero referred to the zero-point energy of the normal molecules. The specific rate of evaporation is then given by introducing the value of $j_{\mathrm{e}}$ from equation 15 into equation 2 . In order to obtain useful results from equation 15 it is necessary to write explicit relations for the partition functions, which can only be done after making definite assumptions concerning the structure of the normal molecules in the condensed state as well as of the structure of the activated molecules formed during evaporation

It will be assumed that the physical state is adequately described by the free volume model. ${ }^{18,19}$ The partition function $Q$ of the normal molecules is then given by the relation

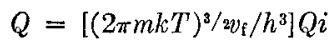

where $Q i$ includes the vibrational, rotational and internal electronic contributions to the partition function, which are assumed to remain unaltered during evaporation. If the partition function $Q^{*}$ corresponding to the activated complex is similarly written as

$$
Q^{*}=\left[(2 \pi m k T) v_{\mathrm{f}}^{2 / 3} / h^{2}\right] Q i
$$

then equation 15 becomes

$$
j_{\mathrm{e}}=K(k T / 2 \pi m)^{1 / 2 v_{\mathrm{f}}}{ }^{-1 / 3} \exp \left(-\cdots \Delta E_{\mathrm{V}} / R T\right)
$$

The values of $j_{\mathrm{e}}$ calculated from equation 18 with $K=1$ are much smaller than the values predicted by the Knudsen equation.

Since the activated complex for evaporation occurs during the formation of freely moving mole-

(18) J. E. Mayer and M. G. Mayer, "Statistical Mechanics," John Wiley and Sons, Inc., New York, N. Y., 1940, pp. 319-326.

(19) E. A. Guggenheim, Proc. Roy. Soc. (London), 135A, 181 (1932) 
cules, it is not unreasonable to postulate that the activated complex consists of gas-like molecules which can move freely throughout a two-dimensional plane of area $v^{* 2 / 3}$ where $v^{*}=v$ is the volume per molecule in the condensed state. ${ }^{3}$ With this assumption equation 15 reduces to the relation

$$
j_{\mathrm{e}}=e K(k T / 2 \pi m)^{1 / 2}\left(v^{2 / 3} / v_{\mathrm{f}}\right) \exp \left(-\Delta H_{\mathrm{V}} / R T\right)
$$

where $\exp \left(-\Delta E_{\mathrm{V}} / R T\right)$ has been replaced by $e$ $\exp \left(-\Delta H_{\mathrm{v}} / R T\right)$

Equation 19 with $e K=1$ has been used ${ }^{3}$ to calculate evaporation rates for a number of liquid compounds and metallic elements by determining the ratio $v^{2 / 3} / v_{\mathrm{f}}$ from the Kincaid-Eyring expres$\operatorname{sion}^{11}$ for the free volume of a liquid. The use of the Kincaid-Eyring free volume formula for metallic elements is justified by the fact that the Lennard-Jones free volume formula ${ }^{20}$ for solids leads to results which are practically identical with numerical values calculated from the Kincaid-Eyring expression at room temperature. ${ }^{21}$ It was found that equation 19 leads to results which are of the same order of magnitude as calculations based on the Knudsen equation. The agreement between the numerical values was observed to be best for the metallic elements and for non-associated spherical liquid molecules where the Kincaid-Eyring formula for the free volume is most likely to be valid. Equation 19 appeared to lead to evaporation rates with a temperature coefficient different from the temperature coefficient of the Knudsen equation.

The results obtained from the theory of absolute reaction rates, although they are perhaps as good as similar applications of this theory to rate processes other than evaporation rates, are not entirely satisfactory. It seems reasonable to suppose that correct application of the theory of absolute reaction rates should lead to an equation equivalent to the Knudsen equation. Failure to obtain this result may indicate an incomplete description of the initial state or of the activated complex as expressed by the respective partition functions. Thus, the description of the condensed state by the free volume model may be inadequate and may be the source of the discrepancies. A comparison of the results of the theory of absolute reaction rates with those obtained from the Knudsen equation, without explicit introduction of partition functions calculated on the basis of an approximate model for the liquid state appears highly desirable.

All references to the approximate description of the condensed state through partition functions can be eliminated from the theoretical relation for the rate of evaporation by proceeding as follows. The introduction of equation 19 into equation 2 leads to the relation

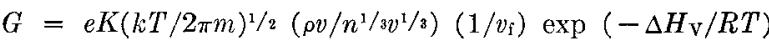

Replacing $\rho v$ by $m$ in this expression for $G$ and replacing $1 / v_{\mathrm{f}}$ by use of equation 9 it is found that

$$
G=e K p_{s}(m / 2 \pi k T)^{1 / 2}
$$

since the product $(n v)^{1 / 3}=1$ as noted earlier in this discussion. Equation 20 is the Knudsen equation for the isothermal rate of evaporation with the evap-

(20) J. E. Lennard-Jones, Proc. Roy. Soc., 52, 729 (1940).

(21 S. S. Penner, J. Chem. Phys., 16, 745 (1948). oration coefficient $\epsilon$ replaced by the product of the base of the natural logarithm $e$ and the transmission coefficient $K .^{3}$

An alternate derivation of equation 20 can be given by noting ${ }^{2}$ that

$$
j_{\mathrm{e}}=K(k T / 2 \pi m)^{1 / 2}(1 / \delta)\left(Q^{* \prime} / Q\right)
$$

where $\delta$ is the length of the energy barrier over which the activated complex passes during evaporation and $Q^{* \prime}$ is the complete partition function of the activated complex. The introduction of equation 21 into equation 2 leads to the result

$$
G=K(k T / 2 \pi m)^{1 / 2}\left(\rho / n^{1 / 3 \delta}\right)\left(Q^{* \prime} / Q\right)
$$

or

$$
G=K(m k T / 2 \pi)^{1 / 2}\left(1 / v n^{1 / 3 \delta}\right)\left(Q^{* \prime} / Q\right)
$$

From equation 9 it can be seen that

$$
p_{\mathrm{s}} v^{2 / 3 \delta} / k T=\left(\delta v^{2 / s} / v_{\mathrm{f}}\right) \exp \left(-\Delta H_{\mathrm{V}} / R T\right)
$$

But if the activated complex behaves as a gaseous molecule and the vibrational, rotational and internal contributions to the partition function remain unchanged during evaporation, then it is evident that

$Q^{* \prime} / Q=e\left(\delta v^{2 / 3} / v_{\mathrm{f}}\right) \exp \left(-\Delta H_{\mathrm{V}} / R T\right)=e p_{\mathrm{s}} \delta v^{2 / 3} / k T$

Replacing $Q^{* \prime} / Q$ by $e \delta p_{\mathrm{s}} v^{2 / \mathrm{a}} / k T$ in equation 22 , it follows that

$$
G=e K p_{\mathbf{s}}(m / 2 \pi k T)^{1 / 2}
$$

It is evident that results calculated from equation 20 with $e K=1$ should be identical with results calculated from the Knudsen equation with the evaporation coefficient set equal to unity. It is therefore apparent that the discrepancies observed between the results calculated from equation 19 with $e K=1$ and from equation 8 with $\epsilon=1$ were introduced by an incomplete description of the physical state through partition functions calculated on the basis of the free volume model. ${ }^{3}$

\section{Application of the Non-Equilibrium Theory of Absolute Reaction Rates to Evaporation Kinetics}

The derivation of equation 20

$$
G=e K p_{\mathrm{s}}(m / 2 \pi k T)^{1 / 2}
$$

seems to be a straightforward result of the application of the usual theory of absolute reaction rates together with the assumptions that the activated molecules are gas-like molecules and that the vibrational, rotational and internal electronic contributions to the partition function remain unchanged during evaporation. Equation 20 cannot be a correct relation for the isothermal rate of evaporation since the maximum possible rate with $K=1$ would be $e$ times as large as the actual upper limit for the isothermal evaporation rate given through equation 8 with $\epsilon=1$. Equations 20 and 8 do not lead to the same maximum values for the isothermal rates of evaporation unless $K$ is restricted to values less than or equal to $1 / e$. This limitation on the numerical values of $K$ seems to be quite unreasonable and can probably not be justified by a more detailed analysis of the transmission coefficient for evaporation. Whatever the relation between $K$ and $\epsilon$ may be, it is apparent that the theory of absolute reaction rates does not lead to the correct upper limit 
for the rate of evaporation unless the factor $e K$ can be replaced by the factor $K$.

The derivation of equation 20 , as well as most other applications of the theory of absolute reaction rates, involves the implicit assumption that equilibrium exists between normal and activated molecules. If this equilibrium does not exist, then the usual theory of absolute reaction rates will lead to evaporation rates which are too large. $9,22,23$ The existence of non-equilibrium can be established for the case at hand by a reductio ad absurdum which leads to the conclusion that the energy barrier $\delta$ must be excessively long if equilibrium does exist between normal and activated molecules.

If equilibrium between normal and activated molecules did exist, then

$$
-k T \ln \left(Q^{* \prime} / Q\right)=p_{\mathrm{s}}\left(v^{*}-v_{i}\right)
$$

Replacing $Q^{* \prime}$ and $Q$ by their respective values leads to the relation

$$
-k T\left[\left(-\Delta E_{\mathrm{V}} / R T\right)+\ln \left(\delta v^{* 2 / 3} / v_{\mathrm{f}}\right)\right]=p_{\mathrm{g}}\left(v^{*}-v_{\mathrm{f}}\right)
$$

Since $v^{*}<<v_{\mathrm{g}}$, it follows that

$$
\Delta H_{\mathrm{V}}-\left[\Delta E_{\mathrm{V}}-N p_{\mathrm{a}}\left(v^{*}-v_{\mathrm{f}}\right)\right] \simeq R T
$$

or

$$
\Delta E_{\mathrm{V}}-N p_{\mathrm{s}}\left(v^{*}-v_{\mathrm{f}}\right) \simeq \Delta E_{\mathrm{V}}
$$

where $N$ is the Avogadro number. Therefore

$$
e \delta v^{* 2 / 3}=e v_{\mathrm{f}} \exp \left(\Delta E_{\mathrm{V}} / R T\right)=v_{\mathrm{f}} \exp \left(\Delta H_{\mathrm{V}} / R T\right)
$$

But from equation 9

$$
v_{\mathrm{g}}=v_{\mathrm{i}} \exp \left(\Delta H_{\mathrm{V}} / R T\right)
$$

The existence of equilibrium between normal and activated molecules therefore leads to the requirement

$$
\delta / v^{* 1 / 3}=(1 / e)\left(v_{g} / v^{*}\right)
$$

i.e., the length of the energy barrier must be equal to about 100 molecular diameters. This result is certainly incorrect and it therefore seems reasonable to conclude that equilibrium between normal and activated molecules cannot exist during evaporation if the gas-like model is a valid description of the activated state.

The mechanism assumed for evaporation seems to be in agreement with the supposition that the molecules move classically in the degree of freedom along which the molecules decompose with an average energy change of the order of $k T$ between successive transfers of energy. Hirschfelder ${ }^{9}$ has shown that in this case the equilibrium theory of reaction rates gives values which are too large by a factor of $1 / 0.387$. The rate treatment for evaporation with Hirschfelder's correction factor therefore leads to the result

$$
G=0.387 e K p_{\mathrm{s}}(m / 2 \pi k T)^{1 / 2}
$$

or

$$
G \simeq K p_{\mathbf{s}}(m / 2 \pi k T)^{1 / 2}
$$

A comparison of equations 8 and 24 indicates that the non-equilibrium theory of absolute reaction rates leads to a result formally identical with the Knudsen equation except that the evaporation co-

(22) H. A. Kramers, Physica, 7, 284 (1940).

(23) B. J. Zwolinski and H. Eyring, J. Am. Chem. Soc., 69, 270 (1947). efficient $\epsilon$ is replaced by the transmission coefficient $K$. Since $\epsilon$ is known to be very close to unity for pure liquids with spherically symmetric force fields (e.g., $\mathrm{Hg}, \mathrm{CCl}_{4}$ ) it follows that $K=1$ in order to obtain agreement between theory and experiment. There are no obvious reasons for not setting the transmission coefficient equal to unity.

\section{Evaporation of Associated Liquids with Hindered Rotation}

The preceding discussion has been restricted to spherically symmetric molecules because equation 9 does not hold for liquids with hindered rotation. In fact, as was emphasized by Kincaid and Eyring, ${ }^{11}$ for liquids with hindered rotation, equation 9 should be replaced by the relation

$$
\frac{1}{v_{\mathrm{f}} \varphi}=\frac{p_{\mathrm{s}}}{k T} \exp \left(\Delta H_{\mathrm{V}} / R T\right)
$$

where $\varphi$ represents the free-angle ratio, i.e., the ratio of the rotational partition function in the liquid to the rotational partition function of the gas. Without introducing any additional assumptions, we may replace the complete internal partition function by the relation

$$
Q i=\varphi Q_{\mathrm{rot}}{ }^{\circ} Q_{\mathrm{vibr} .} Q_{\mathrm{el}}
$$

where $Q_{\text {rot }}{ }^{\circ}$ represents the rotational partition func tion of the gas, and $Q_{\text {vibr. }}$ and $Q_{\mathrm{el}}$ are the vibrational and electronic partition functions, respectively, of the liquid molecules. After division by $e$ to correct for the lack of equilibrium between normal and activated molecules, equation 19 now becomes

$$
j_{\mathrm{e}}=K(k T / 2 \pi m)^{1 / 2}\left(v^{2 / 3} \varphi / v_{i} \varphi\right) \exp \left(-\Delta H_{\mathrm{V}} / R T\right)
$$

After combining equations 25 and 26 and introducing the resulting expression into equation 2 it is found that

$$
G=K \varphi p_{\mathrm{s}}(m / 2 \pi k T)^{1 / 2}
$$

where $K$ would again be expected to be close to unity. Thus a comparison of equations 8 and 27 leads to the conclusion that the evaporation coef-

\begin{tabular}{|c|c|c|c|}
\hline \multicolumn{4}{|c|}{$\begin{array}{c}\text { Comparison between Measured Evaporation } \\
\text { Coefficients } \in \text { and Free-Angle Ratios } \varphi \\
\text { AFTER Wrllie }{ }^{11}\end{array}$} \\
\hline uid & $T,{ }^{\circ} \mathrm{K}$. & $\epsilon$ & $\varphi$ \\
\hline & 273 & 1 & 1 \\
\hline & 279 & 0.90 & 0.85 \\
\hline $\mathrm{Cl}_{3}$ & 275 & .16 & .54 \\
\hline $\mathrm{I}_{5} \mathrm{OH}$ & 273 & .020 & .018 \\
\hline $\mathrm{I}_{3} \mathrm{OH}$ & 273 & .045 & .048 \\
\hline$O_{0}$ & 283 to 303 & 0.036 to 0.040 & .04 \\
\hline
\end{tabular}
ficient for polar liquids should be equal to the freeangle ratio $\varphi$, a result which has been amply confirmed by data published by Wyllie. ${ }^{1}$

In order to emphasize the correlation between $\epsilon$ and $\varphi$ we reproduce Wyllie's compilation in Table I. References to the original literature on the experimental determination of the evaporation coefficient may be found in Wyllie's paper. ${ }^{1}$ The theoretical treatment of evaporation rates given by Wyllie does not permit a clear-cut derivation of equation 27 for the specific evaporation rate of polar liquids.

TABLe I 